\title{
CORRECTION
}

\section{Correction to: One-pot synthesis of hexias (6-0-acryl) cyclodextrin derivatives at room temperature}

\author{
Weirong $\mathrm{Yao}^{2} \cdot$ Shanshan $\mathrm{Xu}^{1} \cdot$ Yanli Cui ${ }^{1} \cdot$ Jianwei Mao ${ }^{3}$
}

Published online: 18 December 2017

๑) Springer Science+Business Media B.V., part of Springer Nature 2017

\section{Correction to: \\ J Incl Phenom Macrocycl Chem (2015) 83:187-191 https://doi.org/10.1007/s10847-015-0505-z}

In the original publication of the article, one of the co-author names Weirong Yao was missed in the author group. And the order of the author group was also changed. These have been updated in this correction article.

The original article can be found online at https://doi. org/10.1007/s10847-015-0505-z.

Yanli Cui

hnzzcyl@hotmail.com

1 Department of Chemistry, Zhejiang University, Hangzhou 310027, China

2 Carbohydrate Chemistry and Biotechnology, Jiangnan University Key Laboratory of Ministry of Education, Wuxi 214122, China

3 Zhejiang Provincial Key Lab for Chem. \& Bio. Processing Technology of Farm Produces, Hangzhou 310023, China 